\title{
REFEREES AMONG MOST IMPORTANT PLAYERS IN SOCCER TOURNAMENTS
}

\author{
Loek Groot
}

Keywords: economics of sports; referee; soccer; bribery

$\mathrm{T}$

he claim I want to put forward is that the referees-and in their wake the linesmen and the fourth officials (assuming that the latter have some role to play)—are among the most important players in tournaments such as the European Championship (EC) and World Championship (WC) football, on an equal footing with the star players entering the pitch. If true, this is not as it should be. Which national team wins should be merely dependent on the performance of the football players. The referee should ideally play a strictly neutral role in this respect, but this is not the case, even if the referee has no preference or interest whatsoever in which team wins. The fact is that he has to make hard decisions during the match, which determines to a large extent the ultimate winner. For this to be true, two conditions have to be met.

First, there is no towering favorite player who beats all opponents during the course of the tournament with a margin of two goals or more. The most recent exception is the Dutch team in 1974 winning all matches with a margin of two goals or more except finally losing against West Germany. Probably more informative is that only 3 of all 40 matches from the quarterfinals onwards on the important tournaments in between 1994 and 2004-the WC ' 94 , the EC ' 96 , the WC ' 98 , the EC 2000, the WC 2002, and EC 2004 (see Tables 1 and 2 below) —end with a difference of more than one goal. Even after extra time because of a draw in the regular time, 37 matches end with a difference of only one goal or are decided by a series of penalties. $E x$ ante, it is therefore not exaggerated to say that whether or not the final champion will reach the final and also wins is a matter of brute luck, depending on how the dice rolls and on the crucial decisions made by the refereeing officials during the course of the tournament.

Second, the referee has to make many decisions during a match in a split second, so even if he intends to be impartial in an impeccable way, he will inevitably have a huge impact on the course and outcome of a match. There are numerous occasions during a match that the referee or linesmen can decide to give the benefit of doubt to one team or the other (e.g., to give

Journal of Sport \& Social Issues, Volume xx, No. x, Month 2005, pp. 1-

DOI: $10.1177 / 0193723505280797$

(C) 2005 Sage Publications 
offside or not, to give a penalty or not, to give a second yellow or red card or not) Even experts in television studios, having seen slow-motion images, often do not agree on whether an action was worth a spot kick or a red card. Therefore, also the second condition is met and the officially impartial officials have sufficiently discretionary power to tip the balance to one side or the other. A recent example is the (un)just disallowed goal of Campbell in the very last minutes of the regular time of the quarterfinal England-Portugal on Euro2004. Now Portugal reached the final, but if the referee would have decided otherwise, England might have been the European champion 2004. What's more, at many corners, there is so much pushing and pulling in front of the goalie that the referee can without any problem decide to give a penalty or disallow a goal. In sum, there are ample opportunities during a match that the referee, or one of the linesmen, can take the discretionary power to bend decisions either way if he wants to. By and large, the often idiosyncratic, with hindsight sometimes bluntly wrong, decisions of referees and linesmen have as great an impact on the outcome of a match (and, because it takes only three matches from the quarterfinals to the final, which team will win the championship) as the performance of the star players on the tournament.

If both conditions are met, the arbitrary quartet also has, in theory at least, the opportunity to let pass one country or the other to the next round, simply by deciding in crucial or dubious cases in the preferred direction. Although, of course, the objectivity and impartiality of the referees are beyond any doubt unless convincing evidence is presented on the contrary, the important role of the arbitrator is a great worry. Referees and linesmen are men of flesh and blood, and the commercial stakes in football are enormous. The WC final of ' 98 was described as a match between Adidas (France) and Nike (Brazil). To minimize the mere chance of bribery, the allocation of officials to matches is crucial. To be sure, in theory, it is possible to grant a strong country the championship only by the help of a few officials: the head of the referee-commission-he is indispensable because he decides ultimately who gets which role in which match-and subsequently in the quarterfinal, the semifinal, and the final, preferably the cooperation of the referee (otherwise a linesman) or, at last resort, the fourth official.

As a rule, potential champions such as France, Germany, Italy, or (at World Championships) Brazil and Argentina will normally survive the first round of the group matches. Only from the quarterfinals onwards the real battles between the giants take place and the knock-out rule is in force. In these confrontations, the outcome can go either way depending on the evaluations of the referees. Tables 1 and 2 present the matches from the quarterfinals onwards of the champion and the team which lost the final of the important tournaments in between 1994 and 2004, with the names of all four officials. Of all these six tournaments there is only one-the EC '96 in England with Germany as winner-in which the complete referee staff was replaced at each successive round of both finalists (see Table 2). At that championship, all four members of the continuously changing refereeing 


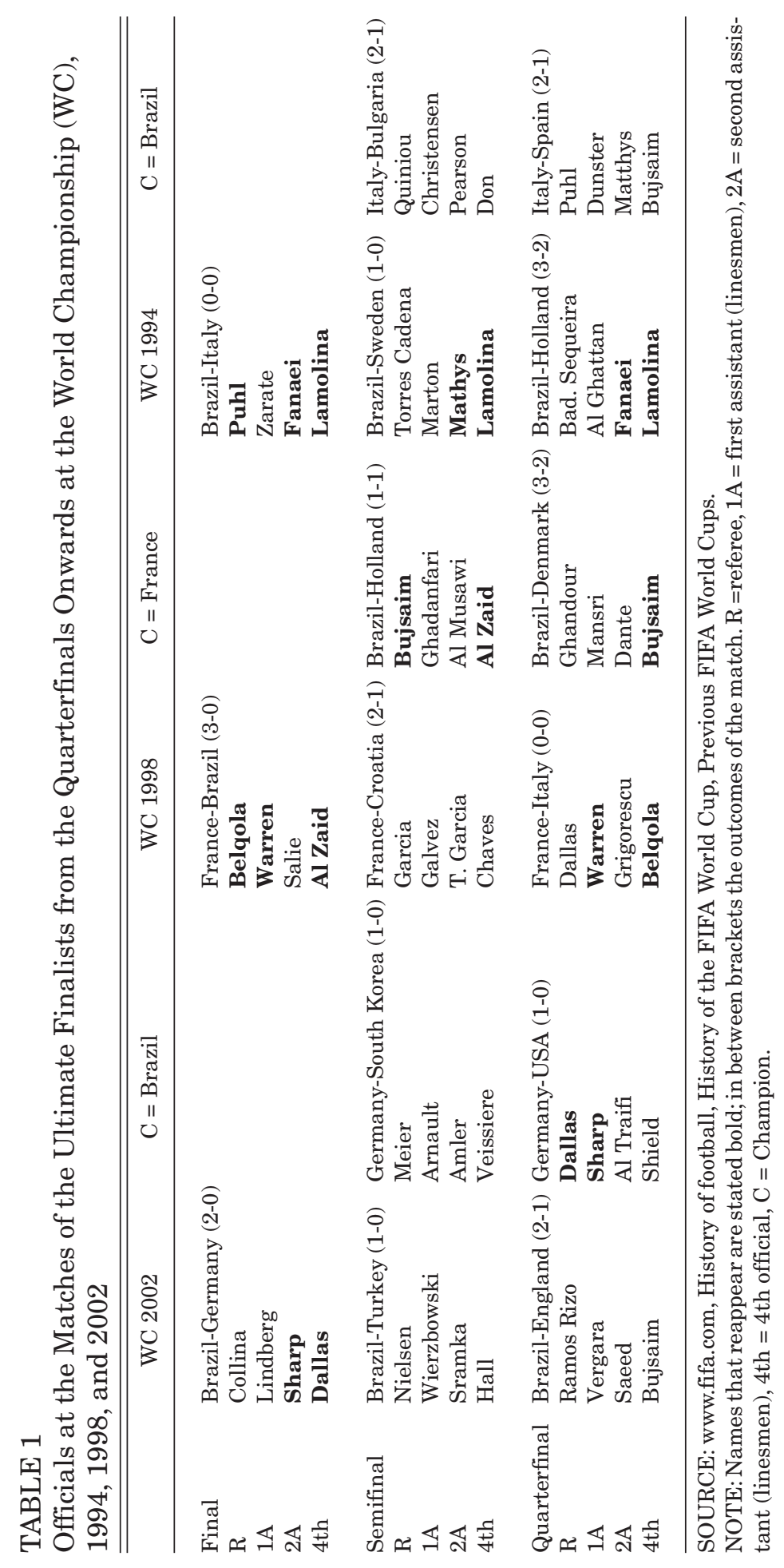




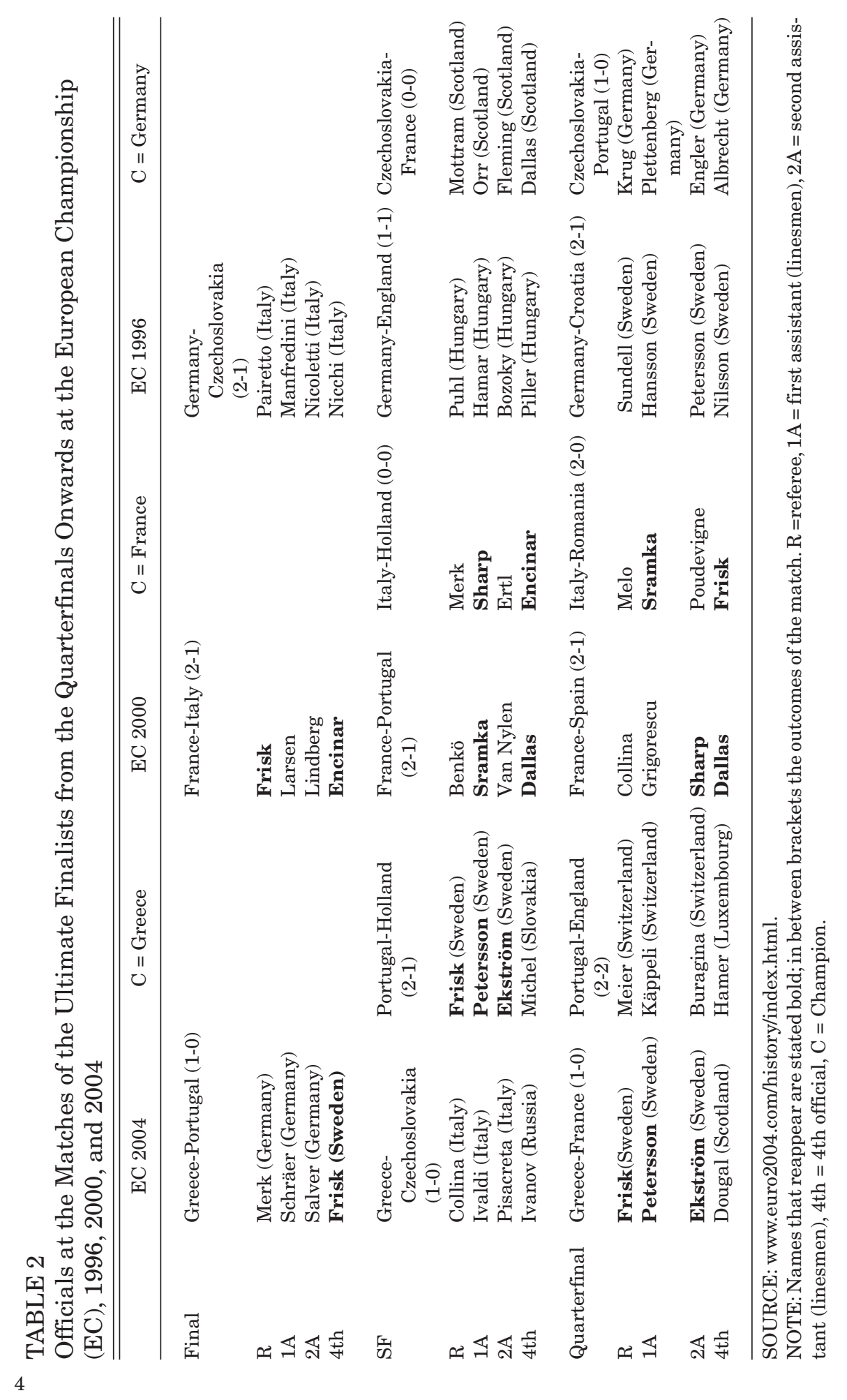


teams came from the same country, in which case it is more difficult to arrange with the help of a few officials a particular outcome of the tournament. At all the other tournaments, some names reappear. For instance, as Table 1 shows, on the WC ' 94 , Lamolina is the fourth official in all matches of Brazil, and Fanaei is linesman in both the quarterfinal against Holland and in the final against Italy. On the WC '98, Warren is involved as linesman in both the quarterfinal and the final of the later champion France. Moreover, Belqola is present in both of these matches, once as fourth official and even as referee in the final.

If one would give any credibility to such a conspiracy theory, then it is not surprising that in the semifinals of Brazil against Sweden on the WC '94 and of France against Croatia on the WC ' 98 , no names reappear. Because both Sweden and Croatia are no leading football countries, the head of the referee commission does not have to select a willing and cooperating member to push the match in a particular direction. With only a small risk the giants, Brazil and France will turn out as winners of these matches. Again, this is pure speculation and only to illustrate how such a conspiracy theory in a minimal fashion would function in practice. However, as the EC '96 shows, it is possible to replace the complete team of officials at each match of the finalists during the last three rounds of a tournament. This practice is more restrictive with respect to the arrangement of a particular result than when the head of the referee commission is in the position to allocate a cooperating official to particular matches whenever it is needed.

However, even if it would be possible to devise a watertight safe procedure for the assignment of truly impartial referees and other officials to matches (e.g., assignment by lottery only a few hours before the match starts), there would inevitably be a large influence of the referee and his assistants on the outcome of the match. One alternative to minimize the impact of referees on the outcome of matches, irrespective of whether conspiracy is a real danger or not, would be to change the rules of the game in such a way that on average, much more, say about 10 , goals are made per match. A possibility that immediately springs to mind would be to enlarge the size of the goal. ${ }^{1}$ If on average, 10 goals are made per match, then one faulty decision will have a fairly limited influence, and many faulty decisions each time to the advantage of the same team becomes readily suspect.

The best way, although this proposal also has a downside, is to make use of television images during the match. Instead of the referee making a ponderous decision in a split second-to give a penalty or not, offside or not, to send a player away or not-the fourth official can, with only a short delay, study the slow-motion replay images and communicate the right decision to the referee. The Union of European Football Association and Fédération Internationale de Football Association have opposed this proposal so far, probably because it would quickly evolve to the situation that on all levels, ranging from the pupils to the veterans, that television or video recording is required to complete a match adequately. The attractiveness of the present, primitive way, with all its shortcomings, is that football is played 
everywhere, from Wembley to the squares in the cities and villages of Ethiopia, with the same means - a football, a field, some lines, and a referee supported by two linesmen; the fourth official has not yet entered the stage below the highest levels.

NOTE

1. Other less obvious reforms, probably with a more modest effect, are imaginable; for instance, that the goalkeeper is not allowed anymore to catch the ball with both hands or more severe punishment of faults committed against attack fielders.

AUTHOR

Loek Groot is economist, philosopher, and currently associate professor at the Department of Public Economics at the Utrecht School of Economics, Utrecht University, The Netherlands. Besides his work on basic income, he wrote several articles on the economics of sports, notably soccer, and on the economics of superstars (see his Web site at http: / / www2.econ.uu.nl/users / l.groot/). 